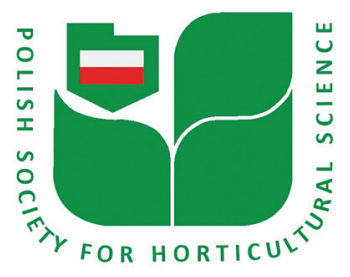

Folia Hort. 32(2) (2020): 279-290

\title{
Screening tea hybrid with abundant anthocyanins and investigating the effect of tea processing on foliar anthocyanins in tea
}

\author{
Jian-Guang Hu', Long-Jie Zhang', Yue-Yue Sheng', Kai-Rong Wang, \\ Yun-Long Shi', Yue-Rong Liang ${ }^{3, *}$, Xin-Qiang Zheng ${ }^{3, *}$
}

${ }^{1}$ Agricultural Technology Extension Center of Yuhang District, Hangzhou 311100, China

${ }^{2}$ Ningbo Huangjinyun Tea Science and Technology Co. Ltd., Yuyao 315412, China

${ }^{3}$ Tea Research Institute, Zhejiang University, Hangzhou 310058, China

${ }^{4}$ Ningbo Agricultural and Rural Bureau, Ningbo 315000, China

\begin{abstract}
Anthocyanins are important bioactive supplements that are consumed from multiple foods and beverage products. Screening tea cultivars producing a high level of anthocyanins can help to enrich the edible bioactive supplements. 'Zijuan' (ZJ) is a tea cultivar growing purple shoots rich in anthocyanins, but it is susceptible to freezing winter and sprouts late in spring. Hybridisation using ' $\mathrm{ZJ}$ ' as the female parent and an early sprouting cultivar 'Wuniuzao' as the male parent was carried out, and four hybrids with purple leaves were obtained. The quality of anthocyanins, catechins, caffeine and amino acids in shoots with three leaves and a bud of the purple leaf hybrids obtained were determined based on the field investigation on sprouting time in spring, winter resistance and leaf yield. It showed that hybrid 'B-2' sprouted earlier in the spring, contained a higher level of anthocyanins and also showed good performance in winter resistance than its female parent ' $\mathrm{ZJ}$ '. It also showed that black tea processing induced a marked decrease in foliar anthocyanins, but green tea processing had little effect on the foliar anthocyanins. Purple tea leaves should be prepared into unfermented green tea instead of fermented black tea to preserve the high level of anthocyanins in the final tea products.
\end{abstract}

Keywords: anthocyanins, black tea, Camellia sinensis, fermentation, green tea, hybrid

Abbreviations: C, catechin; C/A, ratio of catechins to amino acids; GC, catechin gallate; DW, dry weight; EC, epicatechin; ECG, epicatechin gallate; EGCG, epigallocatechin gallate; FW, fresh weight; GCG, gallocatechin gallate; HPLC, highperformance liquid chromatography; WNZ, tea cultivar 'Wuniuzao'; ZJ, tea cultivar 'Zijuan'.

\section{INTRODUCTION}

Anthocyanins, among the polyphenolic constituents, are water-soluble pigments produced as secondary plant metabolites that belong to the flavonoid family of bioactive compounds that give their characteristic red, blue and purple colours to fruits, vegetables and flowers (Saeed et al., 2018; Szpadzik et al., 2019; Gundesli et al., 2019; Gundesli, 2020). Anthocyanidins are widely used as human dietary supplements owing to their bioactivi- ties, such as antimicrobial (Bendokas et al., 2018), antioxidant (Skrzyński et al., 2016), anti-hyperlipidaemia (Qin et al., 2009), anti-hypertension (Naruszewicz et al., 2007), alleviating postprandial inflammation (Jokioja et al., 2020) and anti-metastatic activities (Paramanantham et al., 2020). Anthocyanins are of particular interest to the food industry because of their bioactivity and ability to impart vibrant colours to a variety of food 
products, serving as natural dyes in cyanic colours ranging from salmon pink to red and violet to dark blue. A growing number of clinical evidence suggest that the intake of anthocyanins including their precursor's anthocyanidins at levels equivalent to those present in the recommended daily amounts of fruits and vegetables (i.e., $30-35 \mathrm{mg} \cdot \mathrm{d}^{-1}$ ) may decrease the risk of several age- and obesity-relatedchronic diseases(Pojeretal.,2013; Cassidy et al., 2016; Yang et al., 2017). However, consumption of anthocyanins is still low (Wallace and Giusti, 2019). It was estimated that the daily intake of anthocyanins was $11 \mathrm{mg}$, with about one-third of the population having no intake (Wallace and Giusti, 2019). A finely ground tea powder prepared using tea leaves is now widely used as an antioxidant ingredient in food processing (Sakurai et al., 2017). The tea products rich in anthocyanins will be a potential dietary supplement of anthocyanins. The breeding of tea cultivars with abundant foliar anthocyanins is significant.

The normal tea leaves in green colour contain a low level of anthocyanins. Tea cultivars with purple leaves, owing to their high content of anthocyanins, are increasingly attracting the attention of tea growers and tea consumers (He et al., 2018; Shen et al., 2018; Kilel et al., 2018). However, the available cultivars of purple leaf tea are limited. 'Zijuan' (ZJ) is a tea cultivar with purple leaf from Yunnan province in China where the weather is hot and humid (Bao et al., 2008; He et al., 2018). However, the 'ZJ' has two defects, that is, first, susceptible to freezing winter, resulting in severe freezing-induced injury in winter at high latitude areas; second, late sprouting time in spring, leading to late harvesting of spring tea.

Catechins and amino acids are important indicators for determining the quality potential of a tea cultivar (Jang et al., 2008). Cultivars producing quality black tea usually have a high level of catechins and a higher ratio of catechins to amino acids than those producing quality green tea. During black tea processing, tea catechins are significantly reduced due to oxidation, but information on anthocyanin changes during tea processing has not been available (Luo, 2014). In the current work, a hybridisation using a purple leaf cultivar ' $\mathrm{ZJ}$ ' as female parent and early sprouting and winter resistant cultivar 'Wuniuzao' (WNZ) as male parent was carried out. In the hybrid progenies, four green leaf hybrids and four purple leaf hybrids were obtained. In the obtained purple leaf hybrids, the phenological phase, freezing resistance, leaf yield potential and tea quality-related chemical compositions including anthocyanins, catechins, amino acids and caffeine were determined. Moreover, the changes in foliar anthocyanins during black tea and green tea processing were also investigated. This study aims to screen a winter-resistant and early sprouting hybrid containing a high level of anthocyanins and to investigate the effect of tea processing on foliar anthocyanins in tea leaves.

\section{MATERIALS AND METHODS}

\section{Materials}

\section{Plant materials}

The experiments (Figure 1) were carried out on the Experimental Tea Farm of Zhejiang University, Hangzhou $\left(120.19^{\circ} \mathrm{E}, 30.27^{\circ} \mathrm{N}\right)$, China. Fifty flowers on plants of tea cultivar ' $\mathrm{ZJ}$ ' were artificially pollinated with pollens token from cultivar 'WNZ' in October 2011, and eight seeds were harvested in November 2012. The tea seeds were mixed with water-saturated perlite and placed in an incubator at $4^{\circ} \mathrm{C}$ for 2 weeks and then individually seeded in a plastic pot $(13 \mathrm{~cm}$ in diameter and $15 \mathrm{~cm}$ in height) containing a Pei-Lei organic media (total content of $\mathrm{N}, \mathrm{P}_{2} \mathrm{O}_{5}$ and $\mathrm{K}_{2} \mathrm{O}$ was more than $2 \%$, organic matter was $35 \%, \mathrm{pH} 5.6$ ) purchased from

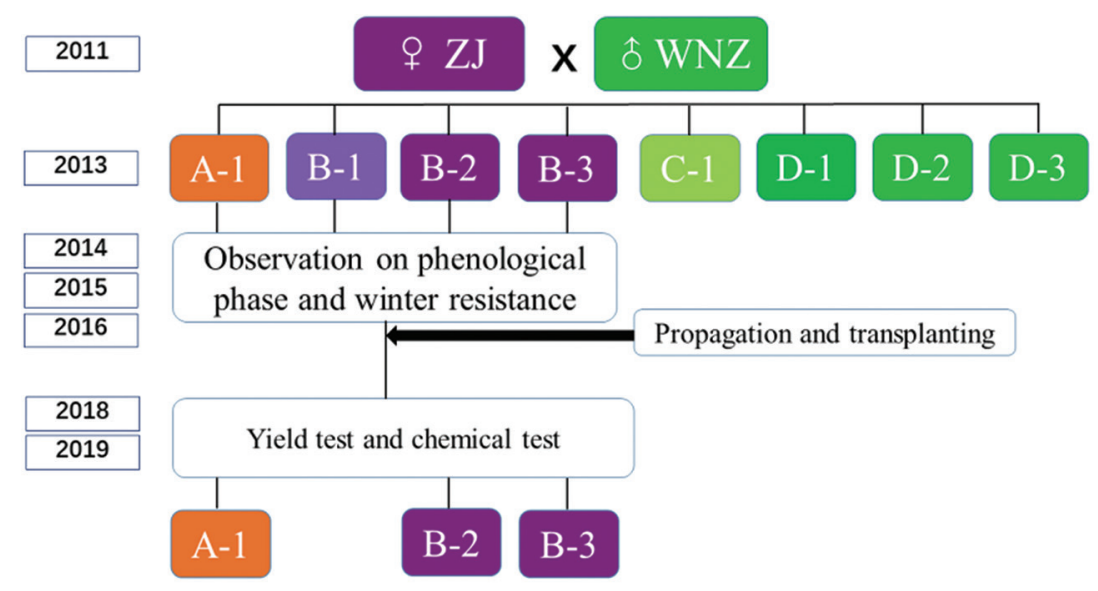

Figure 1. Experimental procedure and the production of tested hybrids. Eight hybrids were obtained by hybridisation with 'ZJ' and 'WNZ'. During 2014-2016, the phenological phase and winter resistance of the hybrids with purple leaves were observed and no further observations of green leaf hybrids were made. 'B-1' was not included in the yield test and chemical test because its sprouting time was later than its female parent ' $\mathrm{ZJ}$ '. 
Zhenjiang Peilei Organic Fertilizer Co., Ltd, Zhenjiang, China). The pots were placed in a growth chamber $(14 \mathrm{~h}$ light at $27^{\circ} \mathrm{C} / 10 \mathrm{~h}$ dark at $22^{\circ} \mathrm{C}, 80 \%$ relative humidity). The plants were transplanted to the field in the Experimental Tea Farm when they grew to more than $30 \mathrm{~cm}$ in height in November 2013. Eight plants were grown from the seeds, of which four grew green leaves and the other four grew purple leaves (Figure 2). The obtained hybrids were classified according to their leaf colour, with 'A' referring to light purple leaf hybrid, 'B' referring to dark purple leaf hybrid, 'C' referring to light green leaf hybrid and ' $\mathrm{D}$ ' referring to deep green leaf hybrid (Figure 2). With early sprouting period in spring and strong winter resistance, this hybridisation aimed to obtain purple leaf cultivar, and thus the phenological phase was investigated on the purple leaf hybrids 'A-1', 'B-1', 'B-2' and 'B-3' and scored according to the scoring system in Figure 3. The dormant overwintered bud was scored 0 point. When the overwintered bud broke dormancy and began to swell, it scored 0.5 point. When the scale leaf was opened, it scored 1.0 point. When the fish leaf was initially opened, it scored 1.5 point. As the fish leaf was fully opened, it scored 2.0 point. The rest was scored by analogy. The observation during 2014-2016 showed that the phenological phase of 'B-1' was later than its female parent 'ZJ' and so it was not observed in the subsequent study. According to the methods by Shi et al. (2019), the winter resistance of the plants was observed after freezing winter in 2016. For further field testing in 2016, tea shoots from hybrids 'A-1', 'B-2' and 'B-3' were harvested for cutting propagation, whereas the late sprouting hybrid ' $\mathrm{B}-1$ ' and the green leaf hybrids 'C-1', 'D-1', 'D-2' and 'D-3' were not further observed (Figure 1).
The young cuttings were transplanted to the field for further leaf yield and chemical composition testing according to randomised block design with three repetitions (Yang et al., 2011). Each block had a width of $1.5 \mathrm{~m}$ and a length of $10 \mathrm{~m}$, in which 60 plants were planted in February 2016. The pruning, fertilising and plucking were carried out according to the requirements of tea cultivar field testing (Yang et al., 2011). In April 2018, shoots with three leaves and a bud were sampled from the plants, wrapped in an aluminium foil, placed in an iced box and taken back to the laboratory where the fresh tea shoots were immediately extracted for HPLC analysis of anthocyanins, catechins and caffeine. The remained tea samples were stored at $-80^{\circ} \mathrm{C}$.

\section{Green tea and black tea}

To confirm the changes in the content of anthocyanins during tea processing, fresh tea shoots with three leaves and a bud were harvested from bushes of purple leaf hybrids 'A-1', 'B-2', 'B-3' and their parents ' $\mathrm{ZJ}$ ' and 'WNZ' in June 2020 and then divided into two portions ( $2 \mathrm{~kg}$ each) for processing green tea and black tea, respectively. Green tea was processed by fixation at $220^{\circ} \mathrm{C}$ for $5 \mathrm{~min}$, rolled in a rolling machine for $30 \mathrm{~min}$ and dried at $110^{\circ} \mathrm{C}$ (Luo, 2014). Black tea was processed by withering at room temperature for $6 \mathrm{~h}$, rolling for $30 \mathrm{~min}$, fermenting at $30^{\circ} \mathrm{C}$ and $90 \%$ relative humidity for $2 \mathrm{~h}$ and drying at $110^{\circ} \mathrm{C}$ (Luo, 2014).

\section{HPLC references}

HPLC references of pelargonidin 3,5-di-O-glucoside, cyanidin 3-O-galactoside, cyanidin 3-O-glucoside, delphinidin, cyanidine, pelargonidin, peonidin, malvidin, gallocatechin (GC), epigallocatechin (EGC), catechin
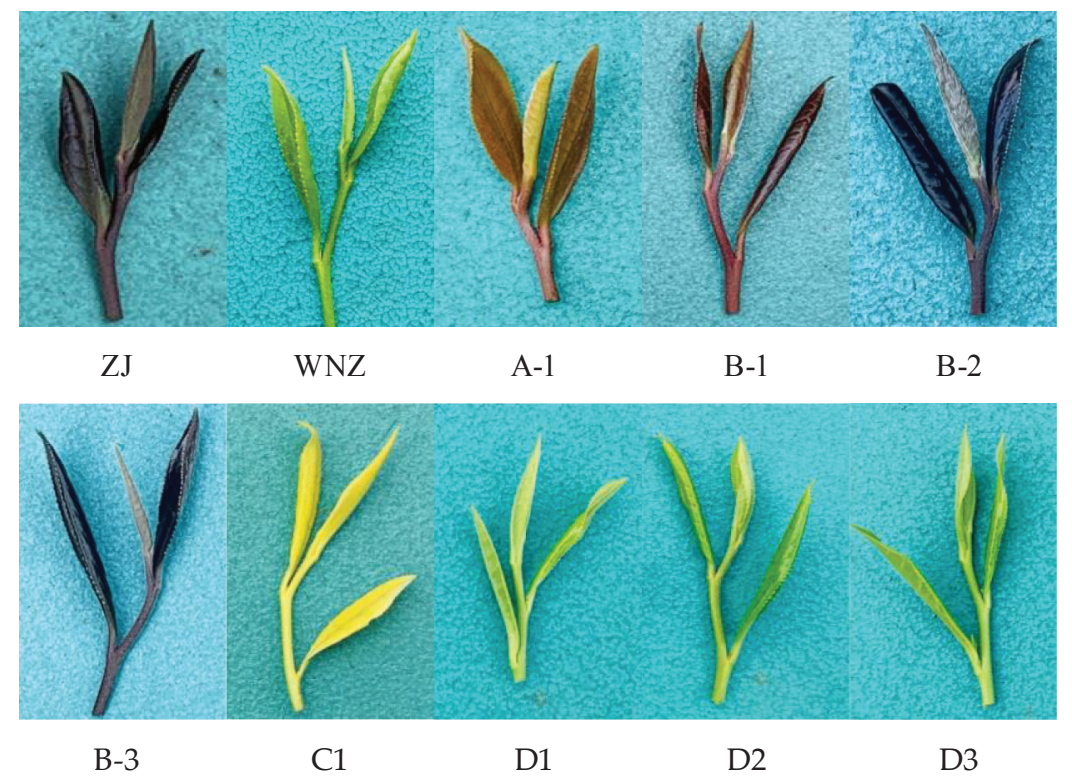

Figure 2. Tea shoot colour of the tested hybrids and their parents. From the hybridisation with female parent ' $\mathrm{ZJ}$ ' and male parent 'WNZ', eight hybrids were obtained, of which four grew green leaves and the other four grew purple leaves. The hybrids were categorised by their leaf colour, with 'A' referring to light purple leaf hybrid, 'B' referring to dark purple leaf hybrid, ' $C$ ' referring to light green leaf hybrid and ' $D$ ' referring to deep green leaf hybrid. 


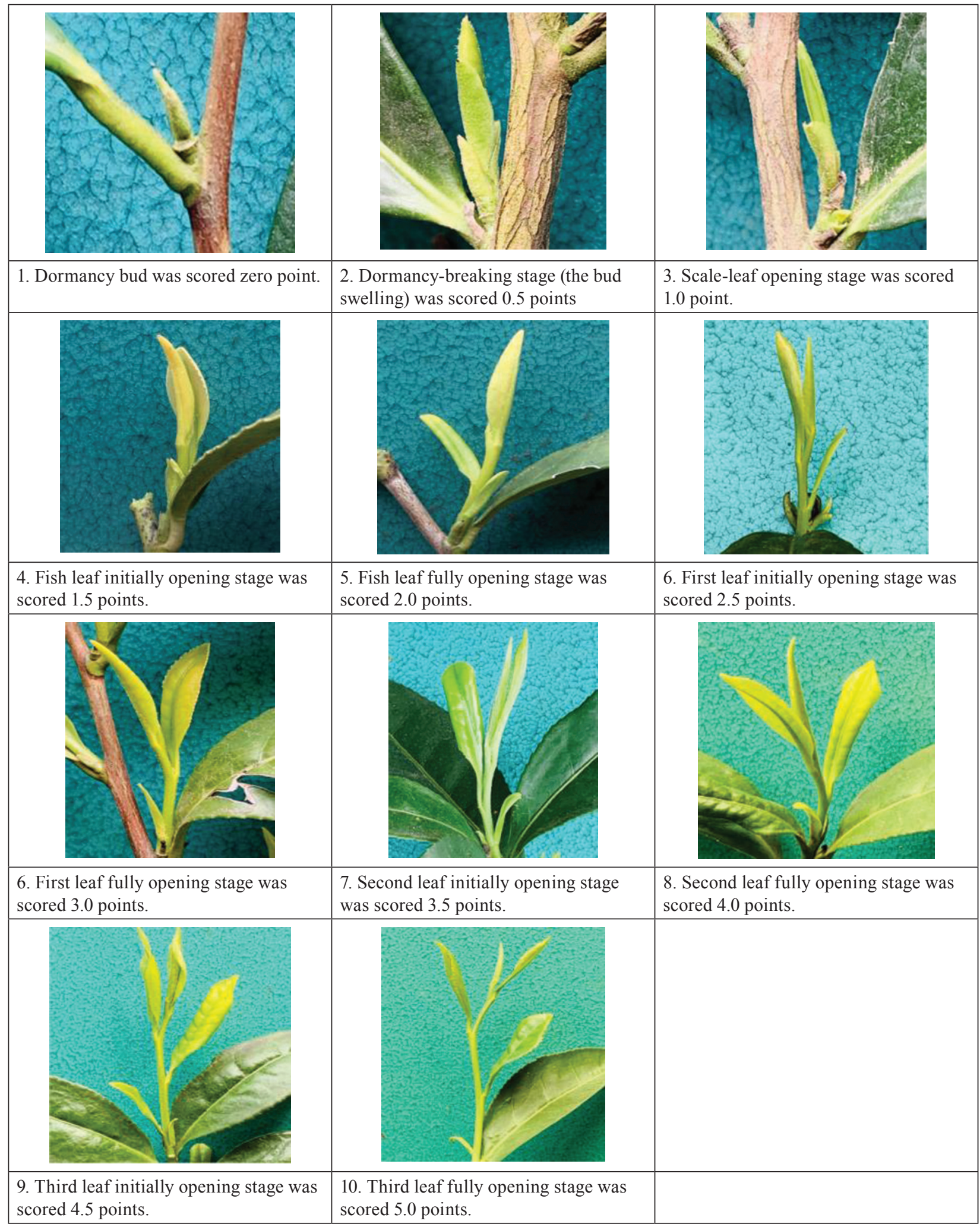

Figure 3. Scoring system for identifying development stages of tea shoots. The dormant overwintered bud was scored Zero point. When the overwintered bud broke dormancy and began to swell, it scored 0.5 point. When the scale leaf was opened, it scored 1.0 point. When the fish leaf was initially opened, it scored 1.5 point and as the fish leaf was fully opened, it scored 2.0 point. The rest was scored by analogy.

(C), epicatechin (EC), epigallocatechin gallate (EGCG), gallocatechin gallate (GCG), epicatechin gallate (ECG), catechin gallate $(\mathrm{CG})$, caffeine, theophylline, theacrine and theobromine were purchased from Sigma-Aldrich
Corporation (St. Louis, USA). The other chemicals used in the present study were HPLC grade reagents purchased from Sinopharm Chemical Reagent Co., Ltd. (Shanghai, China) except for those stated otherwise. 


\section{Methods}

\section{Quantification of anthocyanins}

Tea samples $(100 \mathrm{mg})$ were ground in liquid nitrogen and then extracted in a $5 \mathrm{~mL}$ centrifuge tube containing $2 \mathrm{~mL}$ of extraction solution (methanol: $\mathrm{HCl}=99: 1$ ) at $50^{\circ} \mathrm{C}$ water bath for $30 \mathrm{~min}$, during which the tube was agitated for about $5 \mathrm{~s}$ every $5 \mathrm{~min}$. After centrifugation at $10,000 \times \mathrm{g}$ for $10 \mathrm{~min}$, the supernatant was filtrated through a $0.22 \mu \mathrm{m}$ Millipore filter before being injected into an HPLC system (Shimadzu LC-20AD, Shimadzu Corporation, Kyoto, Japan) equipped with Agilent TC-C18 column $(1.8 \mu \mathrm{m}$, $4.6 \mathrm{~mm} \times 250 \mathrm{~mm}$ ) (Agilent Technologies Inc., Norwood, USA). The injection volume was $10 \mu \mathrm{L}$, and the column temperature was maintained at $35^{\circ} \mathrm{C}$. The elution solvent system consisted of $0.5 \%(\mathrm{v} / \mathrm{v})$ formic acid (solvent A) and acetonitrile (solvent B). Linear gradient elution was programmed from $88 \%(\mathrm{v} / \mathrm{v})$ solvent A to $75 \%$ solvent A in $35 \mathrm{~min}$. The effluent was monitored by UV-vis detector at $520 \mathrm{~nm}$. The identification of the anthocyanins was based on the retention time of the authentic reference compounds. The quantification was carried out by comparing the peak area to be tested with those of the authentic references.

\section{Quantification of catechins and alkaloids}

Tea samples $(250 \mathrm{mg})$ were ground and extracted with $10 \mathrm{~mL} 75 \%(\mathrm{v} / \mathrm{v})$ ethanol for $10 \mathrm{~min}$ and then centrifuged at $10,000 \times g$ at $4^{\circ} \mathrm{C}$ for $15 \mathrm{~min}$. The supernatant was filtrated through a $0.22 \mu \mathrm{m}$ Millipore filter and then injected into HPLC (Jang et al., 2008). Contents of catechins and alkaloids were determined by HPLC (Guclu, 2019; Gundogdu, 2019). The concentrations of catechins and caffeine were determined by comparing the tested peak area with those of the authentic references.

\section{Quantification of amino acids}

Tea samples $(1.00 \mathrm{~g})$ were extracted with $50 \mathrm{~mL}$ of water in a boiling water bath for $30 \mathrm{~min}$ and then filtered on a 'Double-ring' filter paper No 102 (Xinhua Paper Ltd., Hangzhou, China). The total amino acid content was determined using the ninhydrin assay method (Jang et al., 2008).

\section{Data analysis}

The field test was carried out in a randomised block design with three repetitions. The chemical analysis test was carried out with more than two biological duplicates (BD), with two technical duplicates of each BD. The data were expressed as mean \pm SD (standard deviation) on Microsoft Excel 2013. The statistical significance of the mean values between samples was assessed by Tukey HSD test on software IBM SPSS ${ }^{\circledR}$ Statistics 17.0.

\section{RESULTS}

\section{Phenological and productive performances}

The phenological observation shows that the sprouting times of various hybrids and cultivars differentiated in the spring (Table 1). Shoots with a fully opened first

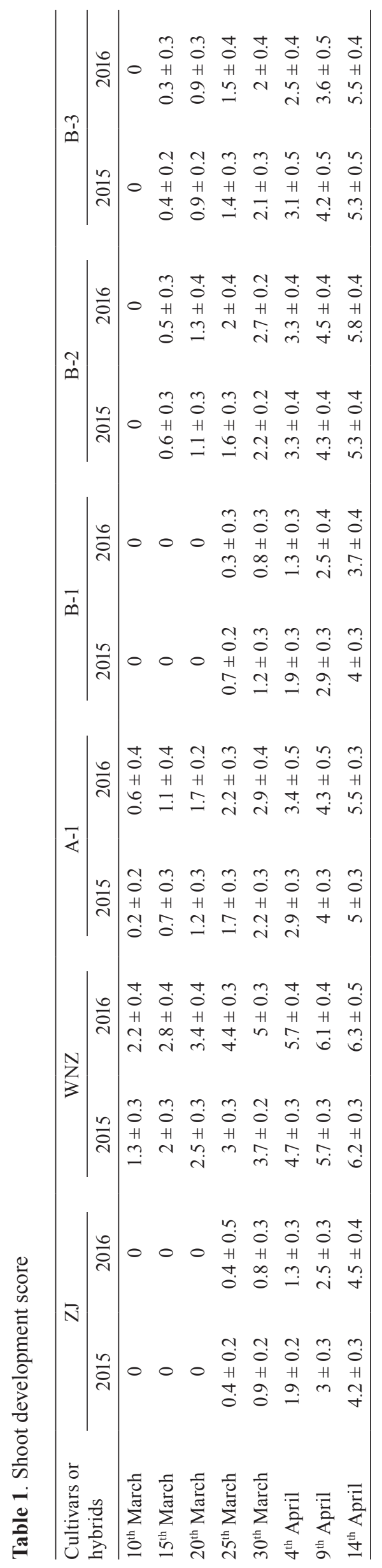


leaf and a bud (shoot development score equal to 3.0 in Figure 3) are usually plucked for processing quality green tea. Based on this plucking standard, the optimum plucking time of ' $\mathrm{WNZ}$ ' was the earliest, and 'B-1' the latest, with 'A-1', 'B-2' and 'B-3' in between. In 2015, the optimum plucking time was March $25^{\text {th }}$ for ' $\mathrm{WNZ}$ ', and it was around April $4^{\text {th }}$ for 'B-2', 'B-3' and 'A-1'. In 2016, the optimum plucking time was around March $20^{\text {th }}$ for 'WNZ', April $4^{\text {th }}$ for ' $\mathrm{B}-2$ ' and April $9^{\text {th }}$ for 'B-3'. Because the phenological phase of 'B-1' was the latest among the tested hybrids, it was not included in the subsequent tests on leaf yield potential and chemical composition (Figure 1).

The lowest temperature, which occurred on $24^{\text {th }}$ January, was $-9^{\circ} \mathrm{C}$ at the Experimental Tea Farm (Hangzhou, China) in 2016. The subsequent winter resistance showed that there were no freezing induced damaged leaves on the plucking tables of the purple leaf hybrids 'A-1', 'B-2' and 'B-3'. However, there was about $5 \%$ freezing induced injured leaves observed on the plucking table of ' $Z J$ '. These suggest that hybrids 'A-1', 'B-2' and 'B-3' had stronger winter resistance than ' $\mathrm{ZJ}$ '.

Leaf yield test showed that ' $\mathrm{ZJ}$ ' had the highest leaf yield in the tested cultivars and hybrids, followed by 'B-2'. 'B-2' had significantly higher leaf yield than 'WNZ'. No significant difference was observed in leaf yield between 'B-2' and ' $\mathrm{ZJ}$ ' in 2019. The leaf yield of 'B-3' was next to 'B-2', and 'A-1' had the least leaf productivity (Table 2 ). These suggest that 'B-2' has good leaf productivity potential.

\section{Variation in contents of anthocyanins}

HPLC test showed that there were 12 peaks detected in the extracts of foliar anthocyanins in the tested hybrids and cultivars (Figure 4), in which four peaks were not identified (peaks A-D in Figure 4) owing to lack of reference compounds and eight were characterised. The identified anthocyanins were pelargonidin 3,5-di-O-glucoside (peak 1), cyanidin 3-O-galactoside (peak 2), cyanidin 3-O-glucoside (peak 3), delphinidin (peak 4), cyanidine (peak 5), pelargonidin (peak 6), peonidin (peak 7) and malvidin (peak 8) (Figure 4).

A quantitative study using authentic compounds as HPLC references showed that the content of anthocyanins varied between hybrids and cultivars. The total content of anthocyanins in 'B-2' was the highest, subsequently followed by 'B-3', ' $\mathrm{ZJ}$ ' and 'A-1', and that of 'WNZ' was the least among the tested cultivars and hybrids (Table 3). The composition of anthocyanins was also differentiated among the hybrids and cultivars. In the identified anthocyanins, pelargonidin 3,5-di-O-glucoside was the most abundant in 'B-2', 'B-3', 'ZJ' and 'WNZ', but malvidin was the most abundant in 'A-1'. Peonidin was the least anthocyanins in all the tested hybrids and cultivars (Table 3).

\section{Variation in catechins and alkaloids}

Catechins and caffeine are important indicators for assessing the quality of tea cultivar. Eight catechins, namely, epicatechin (EC), epicatechin gallate (ECG), epigallocatechin (EGC), epigallocatechin gallate (EGCG), catechin (C), catechin gallate (CG), gallocatechin (GC) and gallocatechin gallate (GCG), were detected in the tested samples. Though authentic caffeine, theophylline, theacrine and theobromine were used as HPLC references (Figure 5) to identify alkaloids

Table 2. Fresh leaf yield $\left(\mathrm{g} \cdot \text { block }^{-1} \text {, mean } \pm \mathrm{SD}\right)^{*}$

\begin{tabular}{cccccc}
\hline Year & \multicolumn{1}{c}{ ZJ } & B-2 & B-3 & WNZ & A-1 \\
\hline 2018 & $2,133.0 \pm 68.5 \mathrm{a}$ & $2,097.7 \pm 56.4 \mathrm{~b}$ & $1,986.7 \pm 60 \mathrm{c}$ & $1,913.7 \pm 75 \mathrm{~d}$ & $1,798.3 \pm 50.4 \mathrm{e}$ \\
2019 & $2,551.3 \pm 43 \mathrm{a}$ & $2,564.3 \pm 39.1 \mathrm{a}$ & $2,415.7 \pm 41.5 \mathrm{~b}$ & $2,316.3 \pm 38.6 \mathrm{c}$ & $2,190.3 \pm 42.5 \mathrm{~d}$ \\
\hline
\end{tabular}

*The data marked with different alphabetic lowercase letters in the same row were significantly different at $p<0.05(n=3)$.

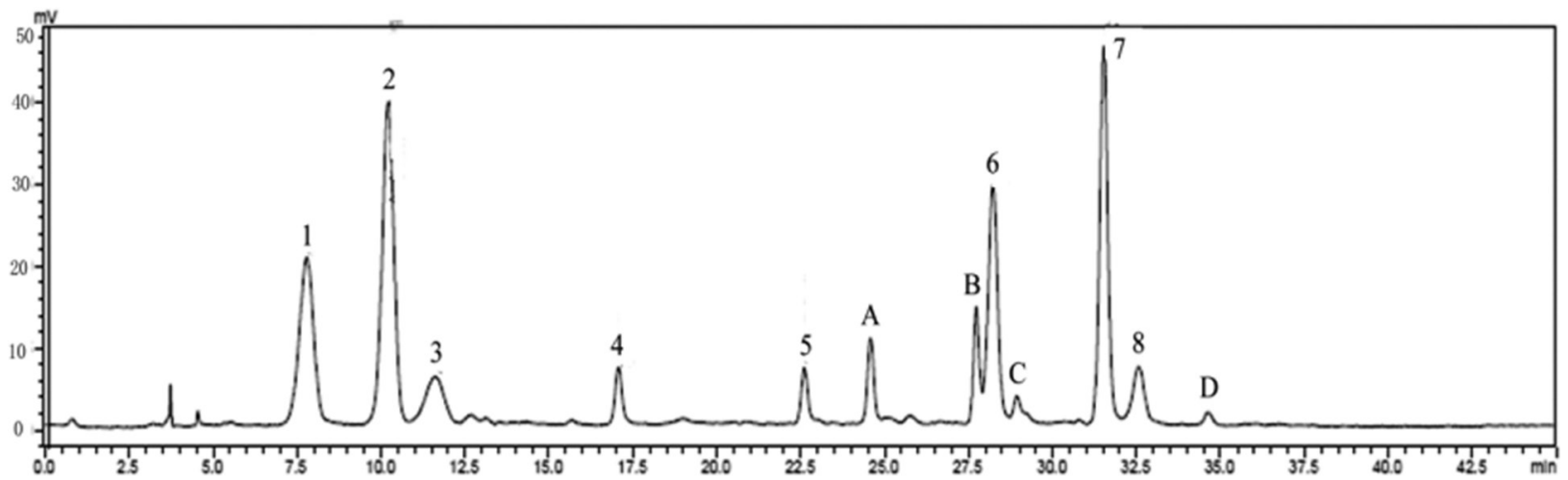

Figure 4. HPLC profile of anthocyanins. 1. Pelargonidin 3,5-di-O-glucoside; 2. cyanidin 3-O-galactoside; 3. cyanidin 3-O-glucoside; 4. delphinidin; 5. cyanidine; 6. pelargonidin; 7. peonidin; 8. malvidin; peaks A-D were not identified owing to lack of reference compounds. 
in the tea samples, only caffeine was detected in the tested tea cultivars or hybrids (Table 4).

EGCG and ECG were the top two abundant catechins and CG was the least among the identified catechins. ' $\mathrm{ZJ}$ ' had the highest level of total content of catechins, followed by 'A-1', and 'B-2' was the least, with 'B-3' and 'WNZ' being ranked three among the tested cultivars and hybrids (Table 4). The content of caffeine was ranged from $8.62 \mathrm{mg} \cdot \mathrm{g}^{-1}$ ('WNZ') to $9.18 \mathrm{mg} \cdot \mathrm{g}^{-1}$ ('ZJ' and 'B-3'), based on fresh weight (FW) (Table 5).

\section{Variation in content of amino acids}

Content of amino acids is a key indicator for assessing the quality of tea, especially for green tea. The content of amino acids ranged from $6.10 \mathrm{mg} \cdot \mathrm{g}^{-1}$ $(\mathrm{FW})$ to $9.80 \mathrm{mg} \cdot \mathrm{g}^{-1}(\mathrm{FW})$, with 'A-1' the highest and 'B-2' the least (Table 5). The ratio of catechins to amino acids (C/A ratio) is an indicator for assessing the quality potential of tea cultivars, in which quality black tea cultivars always have higher C/A ratio than green tea cultivars (Liang et al., 1996). The C/A ratio was 4.8 for 'WNZ' which is used to process green tea, and it was 7.75 for ' $Z J$ ' which is used to process black tea. The C/A ratios of the tested hybrids 'A-1', 'B-2' and 'B-3' ranged from 4.12 to 4.48 , which were significantly lower than ' $\mathrm{ZJ}$ ', suggesting they are suitable for processing green tea.

\section{Effects of tea processing on content of anthocyanins}

Changes in anthocyanins differentiated between green tea processing and black tea processing. There was no significant difference in the total content of anthocyanins between fresh leaf and its corresponding green tea (Figure 6), indicating that green tea processing had little effect on foliar anthocyanins. However, the total content of anthocyanins was significantly decreased during black tea processing, especially in rolling and fermentation stages (Figures 7 and 8). The residue anthocyanins in black tea was $30.94 \%$ for 'B-2', $37.59 \%$ for 'B-3', $36.25 \%$ for 'A-1', $37.54 \%$ for ' $\mathrm{ZJ}$ ' and $76.95 \%$ for ' $\mathrm{WNZ}$ ' compared to their fresh leaves, suggesting that anthocyanins decreased more quickly in anthocyanin abundant leaves than anthocyanin fewer leaves during black tea processing. The loss of anthocyanin during the rolling and fermentation stages accounted for more than $68 \%$ of the total loss of anthocyanin during black tea processing (Figures 7 and 8), suggesting that black tea rolling and fermentation have a great impact on the foliar anthocyanins.

\section{DISCUSSION}

There was a great difference in the content of foliar anthocyanins between tea cultivars. Breeding tea cultivars with abundant foliar anthocyanins is interesting for developing a novel source of anthocyanins dietary 
$\mathrm{mV}$

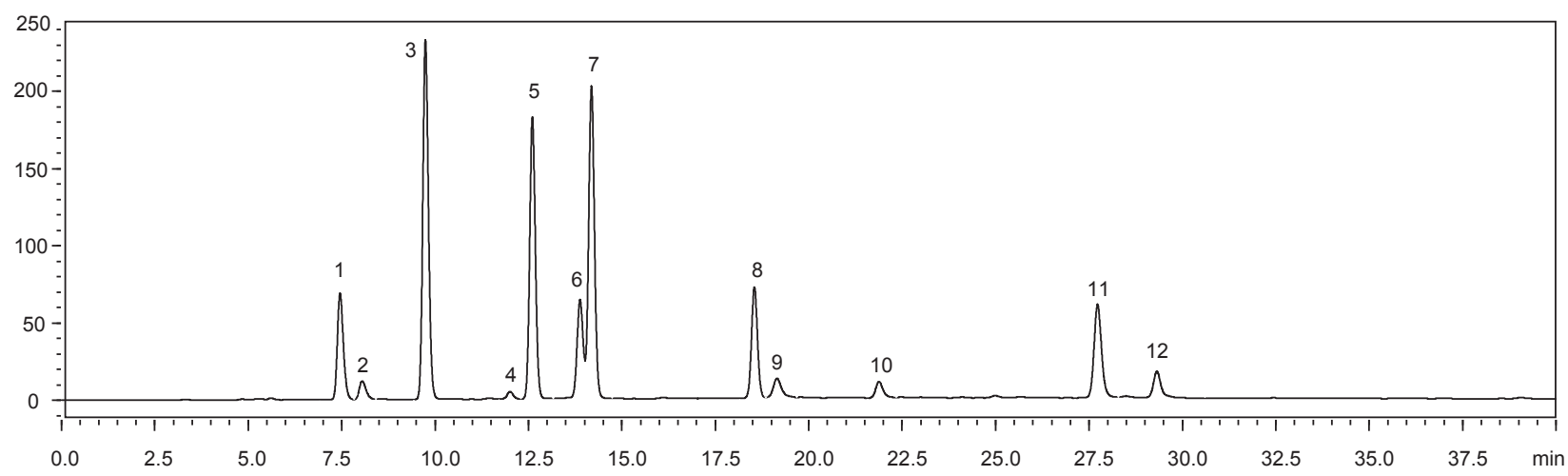

Figure 5. HPLC profile of catechins and caffeine references. 1. theobromine; 2. gallocatechin (GC); 3. theophylline; 4. epigallocatechin (EGC); 5. theacrine; 6. catechin (C); 7. caffeine; 8. epicatechin (EC); 9. epigallocatechin gallate (EGCG); 10. gallocatechin gallate (GCG); 11. epicatechin gallate (ECG); 12. catechin gallate (CG).

supplements. Tea cultivar 'Mooma 1' had $0.5918 \mu \mathrm{g} \cdot \mathrm{g}^{-1}$ DW) of foliar anthocyanins (He et al., 2018). Li et al. (2020) reported that a purple leaf tea cultivar 'Ziyan' had total content of anthocyanins up to $1,079.8 \mu \mathrm{g} \cdot \mathrm{g}^{-1}$ (DW). Purple shoots of cultivar 'Zixin' contained $35 \mu \mathrm{mol} \cdot \mathrm{g}^{-1}$ anthocyanins (equivalent 20,860 $\mu \mathrm{g} \cdot \mathrm{g}^{-1}$ pelargonidin 3,5-di-o-glucoside) (Shen et al., 2018). Tea hybrid 'B-2' obtained in the present study had total content of anthocyanins 4,298.55 $\mu \mathrm{g} \cdot \mathrm{g}^{-1}$ (FW), being 28.8\% higher than ' $\mathrm{ZJ}$ ' (Table 3). Fresh tea shoots with three leaves and a bud usually contain about $75 \%$ moisture (Luo, 2014). Assuming the fresh shoots of 'B-2' contained 75\% moisture, its total content of anthocyanins would be above $17,000 \mu \mathrm{g} \cdot \mathrm{g}^{-1}$ (DW). Exactly, Figure 6 shows that fresh leaves of 'B-2' contained $16,076.4 \mu \mathrm{g} \cdot \mathrm{g}^{-1}$ (DW) of total anthocyanins. Hybrid 'B-2' showed better performance in anthocyanins content, winter resistance and sprouting time than ' $\mathrm{ZJ}$ ', and so it will be a potential candidate for producing anthocyanins rich tea or tea products.

Cultivar ' $Z J$ ' in the present study had 3,336.82 $\mu \mathrm{g} \cdot \mathrm{g}^{-1}$ (FW) (Table 3) or 13,380.81 $\mu \mathrm{g} \cdot \mathrm{g}^{-1}$ (DW) (Figure 6) of total anthocyanins, which was lower than the results $\left(29,140 \mu \mathrm{g} \cdot \mathrm{g}^{-1}\right.$, DW) by Bao et al. (2008), but higher than that $\left(377.13 \mu \mathrm{g} \cdot \mathrm{g}^{-1}\right.$, DW) by Wang et al. (2017). These might be due to the differences in growing environments. Bao et al. (2008) sampled the ' $\mathrm{ZJ}$ ' plants grown in Menghai of Yunnan Province (latitude $21.95^{\circ} \mathrm{N}$, mean altitude 1,700 m), and Wang et al. (2017) sampled their ' $\mathrm{ZJ}$ ' plants grown in Fuzhou of Fujian Province (latitude $26.05^{\circ} \mathrm{N}$, mean altitude $10 \mathrm{~m}$ ). The ' $\mathrm{ZJ}$ ' plants in the present study are grown in Hangzhou of Zhejiang Province (latitude $30.27^{\circ} \mathrm{N}$, mean altitude $20 \mathrm{~m}$ ). Ultraviolet A and ultraviolet B upregulate the expression of structural genes such as F3'5 'H encoding flavonoid 3',5'-hydroxylase, DFR encoding dihydroflavonol 4-reductase and ANS encoding anthocyanidin synthase, as well as regulatory gene TT2 (TRANSPARENT
TESTA 8) in the anthocyanin pathway, resulting in high accumulation of anthocyanins (Li et al., 2020). Ultraviolet radiation is usually stronger at high altitude areas than low altitude areas. This explains why the total content of anthocyanins of ' $\mathrm{ZJ}$ ' in the present study was between those by Bao et al. (2008) and Wang et al. (2017).

Anthocyanins are one of the major contributors to the antioxidant activity of tea and tea products (Lu et al., 2015). The bioactivity of anthocyanins in tea varies with their composition and concentration. The most abundant component of anthocyanins in commercial tea prepared using cultivar ' $\mathrm{ZJ}$ ' in Yunnan was delphinidin 3-O-beta-D-(6-(E)-p-coumaroyl) galactopyranoside (Jiang et al., 2013), while cyanidin 3-O-beta-D-(6-(E)coumaroyl) glucopyranoside showed the highest in vitro antioxidant activity in the leaf of an Indian purple leaf tea cultivar (Joshi et al., 2017). When the same batch of fresh leaves plucked from plants of cultivar 'ZJ' were separated into two portions to be prepared into unfermented green tea and fermented black tea respectively, the black tea showed much lower antioxidant activity than the green tea (Lu et al., 2015), indicating tea processing has a great impact on the antioxidant activity of anthocyanins abundant tea leaf. The present study showed that green tea processing has a little effect on the content of anthocyanins, but black tea processing induced great loss in anthocyanins, especially in the rolling and fermentation stages (Figures 6-8). Black tea fermentation is a process of polyphenol oxidation, in which colourless catechins are oxidised and condensed into thearubigins and theaflavins in red and orange colours. Anthocyanins are a group of polyphenolic compounds. Oxidation of anthocyanins might be an important factor leading to the decrease in total content of anthocyanins during black tea processing. To maintain a high level of anthocyanins in the final tea products, it is considered that the anthocyanin abundant leaves 
Table 5. Content of amino acids and the ratio of catechins to amino acids (mean $\pm \mathrm{SD}$ )*

\begin{tabular}{lcc}
\hline Cultivar & Amino acids $\left(\mathrm{mg} \cdot \mathrm{g}^{-1}, \mathrm{FW}\right)$ & \multicolumn{1}{c}{ C/A ratio } \\
\hline A-1 & $9.80 \pm 0.20 \mathrm{a}$ & $4.47 \pm 0.13 \mathrm{bc}$ \\
$\mathrm{B}-2$ & $6.10 \pm 0.00 \mathrm{c}$ & $4.12 \pm 0.23 \mathrm{c}$ \\
$\mathrm{B}-3$ & $8.00 \pm 0.30 \mathrm{~b}$ & $4.48 \pm 0.04 \mathrm{bc}$ \\
WNZ & $7.30 \pm 0.50 \mathrm{~b}$ & $4.80 \pm 0.03 \mathrm{~b}$ \\
ZJ & $8.40 \pm 0.30 \mathrm{~b}$ & $7.75 \pm 0.14 \mathrm{a}$ \\
\hline
\end{tabular}

*The data marked with different lowercase letters in the same column were significantly different at $p<0.05(n=3)$.

harvested from the purple leaf tea cultivars should be prepared into unfermented green tea instead of fermented black tea.

The present study showed that hybrid 'B-2' had a higher content of anthocyanins than both its parents ' $\mathrm{ZJ}$ ' and 'WNZ' (Table 3). The tea plant is an outcrossing species. This phenomenon suggests that the assortment of different parental alleles was favourable in 'B-2'. However, total catechins content in 'B-2' was significantly lower than ' $\mathrm{ZJ}$ ' and ' $\mathrm{WNZ}$ ' (Table 4). The total content of anthocyanins in 'A-1' and 'B-3' was significantly higher than their male parent 'WNZ', but not significantly different from their female parent ' $\mathrm{ZJ}$ '. Total catechins in 'A-1' were significantly higher than 'WNZ' but significantly lower than 'ZJ' (Tables 3 and 4). The biosynthesis of anthocyanins shares a common phenylpropanoid pathway with catechins (Liu et al., 2019; Wang et al., 2019), in which there may be a substrate competition between anthocyanins biosynthesis and catechins biosynthesis (Liu et al., 2019). It is considered that the high accumulation of anthocyanins in ' $\mathrm{B}-2$ ' might be partially at the expense of catechins because of the substrate competition in the phenylpropanoid pathway.

Catechins and amino acids are important indicators to assess the quality of tea and the suitability of a cultivar for processing green tea or black tea. Usually, tea cultivars for processing black tea have a higher content of catechins and higher ratio of catechins to amino acids (C/A ratio) than those for processing green tea. Instead, green tea cultivars usually have a higher content of amino acids and lower C/A ratio than black tea cultivars (Liang et al., 1996; Jang et al., 2008). Nowadays, tea breeders use these indicators to predict the quality potential of a new tea cultivar for processing black tea or green tea (Liang et al., 1996). 'ZJ', a cultivar for processing black tea, had the highest level of catechins and the highest C/A ratio among the tested cultivars and hybrids. The $\mathrm{C} / \mathrm{A}$ ratio of 'A-1', 'B-2', and 'B-3' was significantly lower than ' $\mathrm{ZJ}$ ', among which 'B-2' was the least (Table 5), suggesting that 'B-2' is suitable for processing green tea, especially for anthocyanidins rich green tea. 


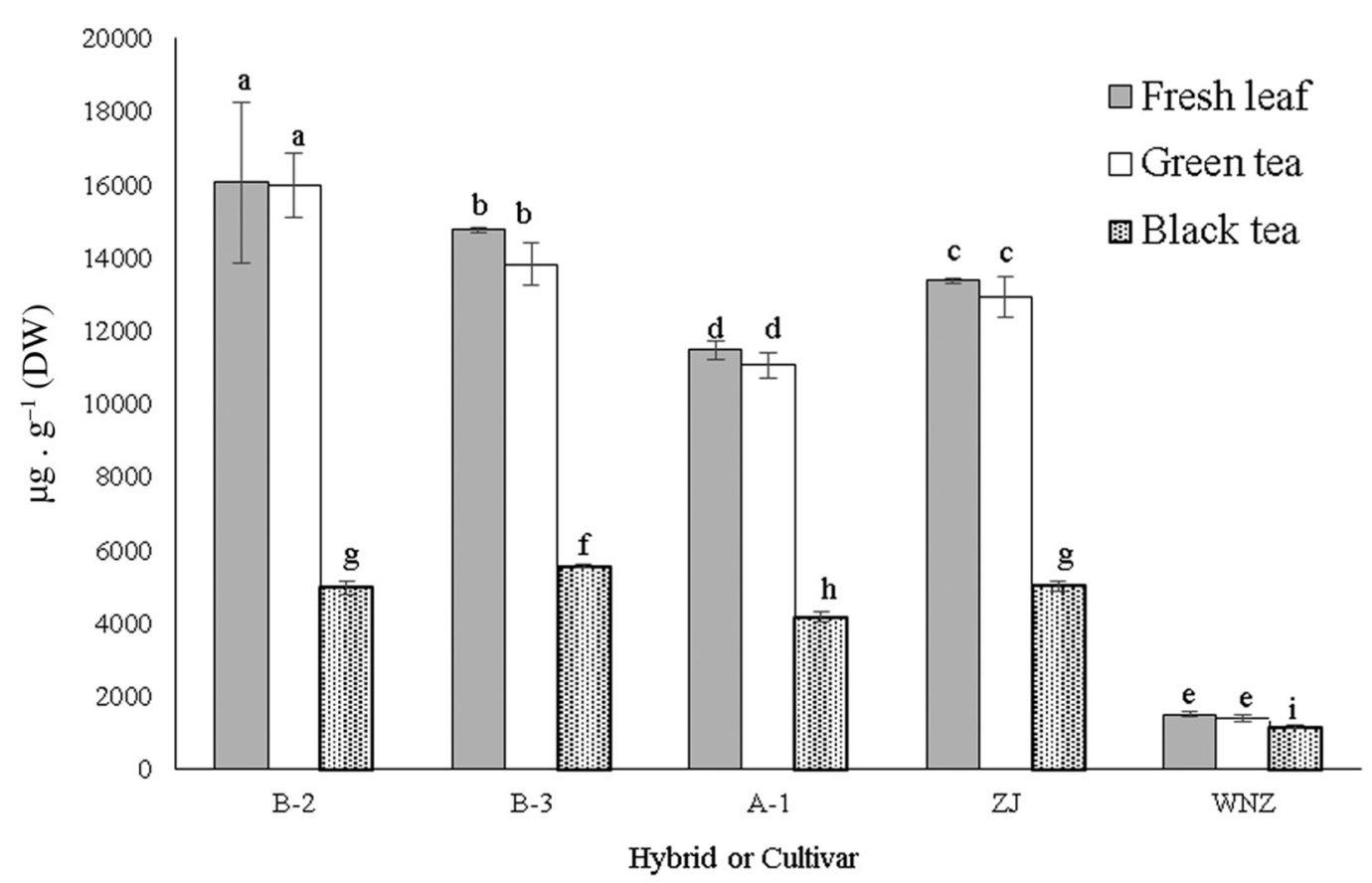

Figure 6. The total content of anthocyanins in fresh leaf, green tea and black tea. The data marked with different lower case alphabetic letters were significantly different at $p<0.05(n=3)$.

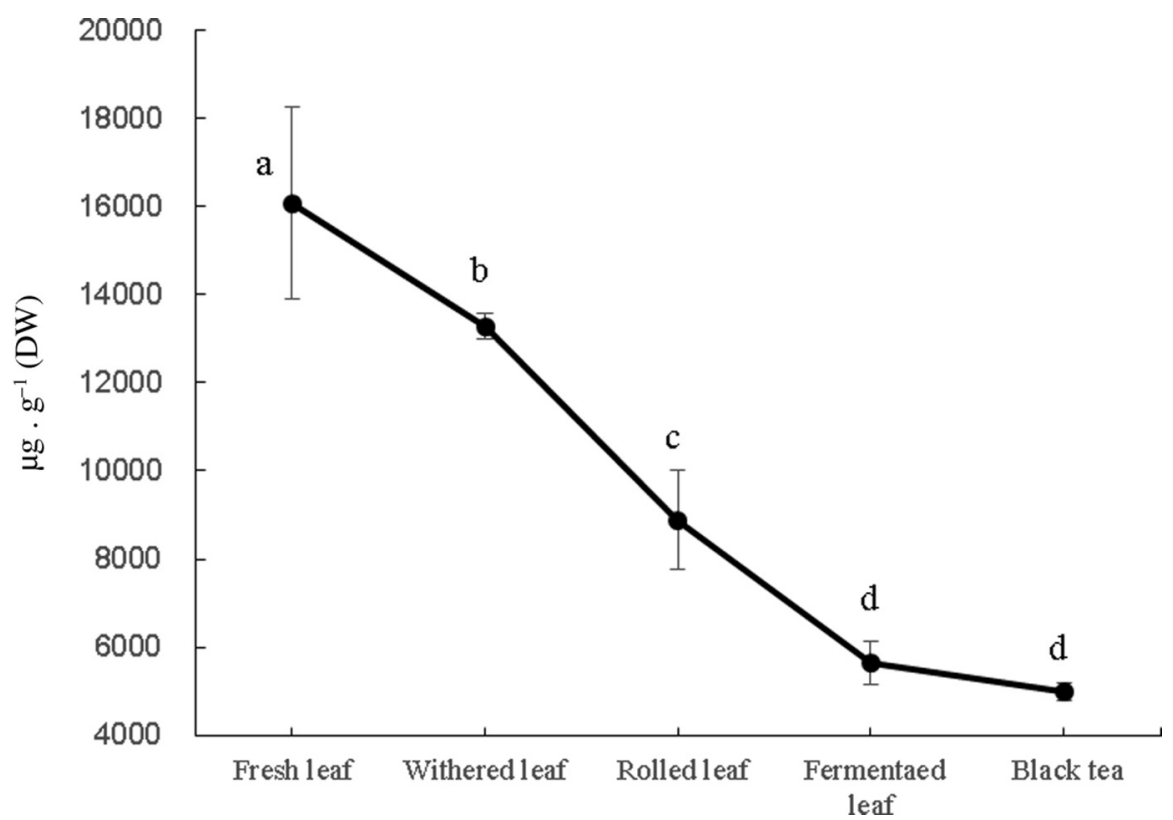

Figure 7. Changes in the total content of anthocyanins during black tea processing using leaves of hybrid 'B-2'. The data marked with different lower case alphabetic letters were significantly different at $p<0.05(n=3)$.

\section{CONCLUSION}

An anthocyanins abundant, early sprouting and winterresistant tea hybrid with purple leaf were obtained. Black tea processing has caused a significant loss of foliar anthocyanins and green tea processing has a little effect on foliar anthocyanins. To maintain a high level of foliar anthocyanins, fresh tea leaves with abundant anthocyanins from purple leaf cultivars should be processed into green tea instead of black tea.

\section{ACKNOWLEDGEMENTS}

The authors appreciated the Science Technology Bureau of Ningbo City and the Science Technology Department of Zhejiang Province for offering financial support to the present project. The authors also appreciated Dr. Jian-Liang Lu, Dr. Jian-Hui Ye, Dr. Qing-Sheng Li, Dr. Ying Wu, Dr. Da Li, Ms. Zhuo-Yu Cai and Mr. Ming Li for their contributions to this project. 


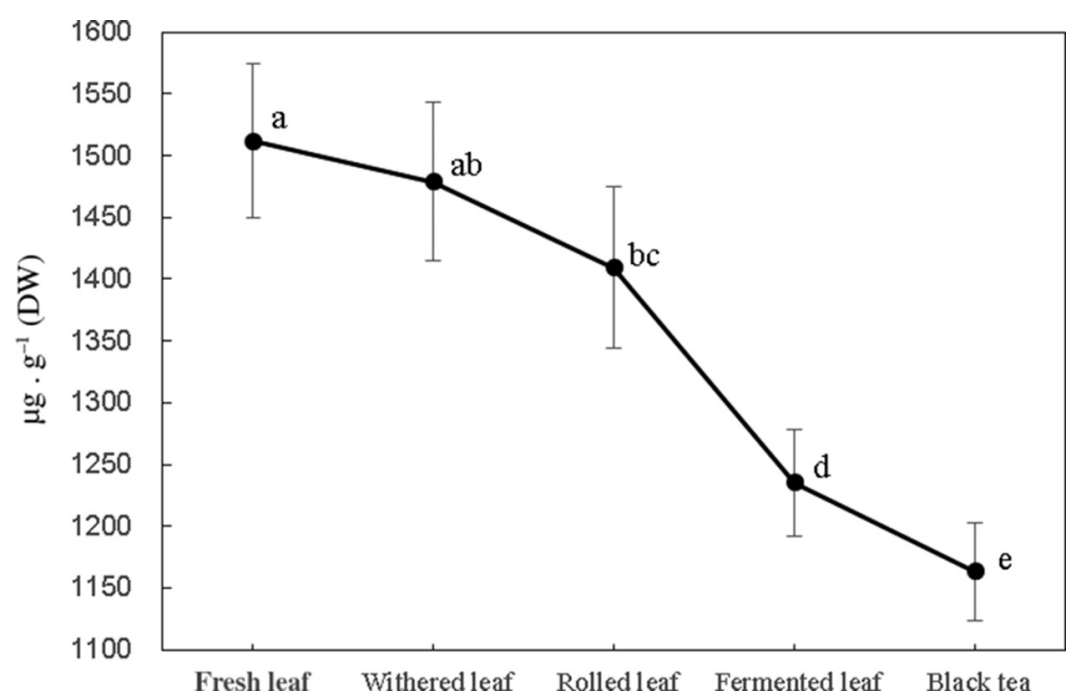

Figure 8. Changes in the total content of anthocyanins during black tea processing using leaves of cultivar 'WNZ'. The data marked with different lower case alphabetic letters were significantly different at $p<0.05(n=3)$.

\section{FUNDING}

This research was financially supported by grants from the Ningbo Municipal Bureau of Science and Technology (Project No. 2017C10001) and the Science Technology Department of Zhejiang Province (Project No. 2016C02053-5), China.

\section{AUTHOR CONTRIBUTIONS}

Y.R.L., K.R.W. and X.Q.Z. conceived the project. J.G.H. performed phenological and leaf yield investigation. L.-J.Z. and K.-R.W. generated the field experiments and prepared leaf samples. Y.-Y.S. and Y.-L.S. performed chemical analysis. Y.R.L., L.J.Z., J.G.H., Y.Y.S., X.Q.Z. and K.R.W. prepared the manuscript. All authors read the final manuscript and approved the submission.

\section{CONFLICTS OF INTEREST}

All of the authors declare no conflict of interest.

\section{REFERENCES}

BAO, Y. X., XIA, L. F., Li, Y. Y., AND LiAnG, M. Z. (2008). A new tea tree cultivar 'Zijuan'. Acta Horticulturae Sinica, 35, 934 (in Chinese).

Bendokas, V., Šarkinas, A., Jasinauskiené, D., AnisimovienĖ, N., MORKŪNAITE்-Haimi, $\breve{S}$., StANYS, V., AND ŠSIKŠnIANAS, T. (2018). Antimicrobial activity of berries extracts of four Ribes species, their phenolic content and anthocyanin composition. Folia Horticulturae, 30(2), 249-257.

Cassidy, A., Bertoia, M., Chiuve, S., Flint, A., Forman, J., AND Rimm, E. B. (2016). Habitual intake of anthocyanins and flavanones and risk of cardiovascular disease in men. American Journal of Clinical Nutrition, 104, 587-594.

GuClu, S. F. (2019). Identification of polyphenols in homogenetic and heterogenetic combination of cherry graftings. Pakistan Journal of Botany, 51, 2067-2072.

GuNDESLI, M. A. (2020). Determination of sugar contents, Total phenol and antioxidant activity of various parts 'Uzun' pistachio cultivar (Pistacia vera L.). International Journal of Agriculture Environment and Food Sciences, 4(1), 52-58.

Gundeşli, M. A., Korkmaz, N., and Okatan, V. (2019). Polyphenol content and antioxidant capacity of berries: A review. International Journal of Agriculture Forestry and Life Sciences, 3(2), 350-361.

Gundogdu, M. (2019). Effect of rootstocks on phytochemical properties of apricot fruit. Turkish Journal of Agriculture and Forestry, 43(1), 1-10.

He, X. J., Zhao, X. C., Gao, L. P., Shi, X. X., Dai, X. L., LiU, Y. J., XIA, T., AND WANG, Y. S. (2018). Isolation and characterization of key genes that promote flavonoid accumulation in purple-leaf tea (Camellia sinensis L.). Scientific Reports, 8, 130, doi: 10.1038/ s41598-017-18133-z.

JANG, J. H., Kim, E. S., Wu, S. Y., Lu J. L., Liang, H. L., Du, Y. Y., Lin, C., AND Liang, Y. R. (2008). Assessing geographic origins of green teas using instruments. Food Science and Biotechnology, 17(5), 1016-1020.

Jiang, L. H., Shen, X. J., Shou, T., Kanda, T., Zhou, J. C., AND ZHAO, L. M. (2013). Characterization and activity of anthocyanins in Zijuan tea (Camellia sinensis var. kitamura). Journal of Agricultural and Food Chemistry, 61, 3306-3310.

Jokioja, J.,Linderborg, K.M.,Kortesniemi,M., NuORA,A., Heinonen, J., Sainio, T., Vittanen, M., Kallio, H., AND YANG, B. R. (2020). Anthocyanin-rich extract from purple potatoes decreases postprandial glycemic response and affects inflammation markers in healthy men. Food Chemistry, 310, 125797, doi: 10.1016/j.foodchem.2019.125797.

Joshi, R., Rana, A., Kumar, V., Kumar, D., Padwad, Y. S., Yadav, S. K., And Gulati, A. (2017). Anthocyanins 
enriched purple tea exhibits antioxidant, immunostimulatory and anticancer activities. Journal of Food Science and Technology-Mysore. 54, 1953-1963.

Kilel, E. C., Wanyoko, J. K., Faraj, A. K., and Ngoda, P. (2018). Optimization of manufacturing conditions of the new purple leafed Kenyan teas (TRFK 306) - maceration style and withering duration. International Food Research Journal, 25, 730-736.

Li, W., Tan, L., Zou, Y., Tan, X., Huang, J., Chen, W., AND TANG, Q. (2020). The effects of ultraviolet $\mathrm{A} / \mathrm{B}$ treatments on anthocyanin accumulation and gene expression in dark-purple tea cultivar 'Ziyan' (Camellia sinensis). Molecules, 25, 354, doi: 10.3390/ molecules25020354.

Liang, Y. R., Lu, J. L., And Shang, S. L. (1996). Effect of gibberellins on chemical composition and quality of tea (Camellia sinensis L). Journal of the Science of Food and Agriculture, 72, 411-414.

Liu, Y., Jiang, H., Zhao, Y., Li, X., Dai, X., Zhuang, J., Zhu, M., Jiang, X., Wang, P., GaO, L., and Xia, T. (2019). Three Camellia sinnensis glutathione S-transferases are involved in the storage of anthocyanins, flavonols, and proanthocyanidins. Planta, 250, 1163-1175.

Lu, H. P., Dai, W. D., TAN, J. F., Guo, L., Zhu, Y., And Lin, Z. (2015). Identification of the anthocyanins from the purple leaf coloured tea cultivar Zijuan (Camellia sinensis var. assamica) and characterization of their antioxidant activities. Journal of Functional Foods, 17, 449-458.

Luo, Y. P. (2014). Cultivation and processing of famous teas. Beijing, China: China Agriculture Press Co., Ltd. (pp. 185-197) (in Chinese).

Naruszewicz, M., Łaniewska, I., Millo, B., AND DŁuŻNiewski, M. (2007). Combination therapy of statin with flavonoids rich extract from chokeberry fruits enhanced reduction in cardiovascular risk markers in patients after myocardial infarction (MI). Atherosclerosis, 194, e179-e184.

Paramanantham, A., Kim, M. J., Jung, E. J., Nagappan, A., Yun, J. W., Kim, H. J., Shin, S. C., Kim, G. S., And LeE, W. S. (2020). Pretreatment of anthocyanin from the fruit of Vitis coignetiae Pulliat acts as a potent inhibitor of TNF- $\alpha$ effect by inhibiting NF- $\kappa$ B-regulated genes in human breast cancer cells. Molecules, 25(10), 2396, doi: 10.3390/ molecules25102396.

Pojer, E., Mattivi, F., Johnson, D., and Stockley, C. S. (2013). The case for anthocyanin consumption to promote human health: A review. Comprehensive Reviews in Food Science and Food Safety, 12, 483-508.

Qin, Y., Xia, M., MA, J., HaO, Y. T., Liu, J., Mou, H. Y., CAO, L., AND Ling, W. (2009). Anthocyanin supplementation improves serum LDL- and HDLcholesterol concentrations associated with the inhibition of cholesteryl ester transfer protein in dyslipidemic subjects. American Journal of Clinical Nutrition, 90, 485-492.

Saeed, A., Kauser, S., and Iqbal, M. (2018). Nutrient, mineral, antioxidant, and anthocyanin profiles of different cultivars of Syzygium cumini (jamun) at different stages of fruit maturation. Pakistan Journal of Botany, 50, 1791-1804.

Sakurai, Y., Mise, R., Kimura, S., Noguchi, S., Iwao, Y., AND ITAI, S. (2017). Novel method for improving the water dispersibility and flowability of fine green tea powder using a fluidized bed granulator. Journal of Food Engineering, 206, 118-124.

Shen, J.Z., Zou, Z. W., Zhang, X.Z., Zhou, L., WAnG, Y.H., FAnG, W. P., AND ZHU, X. J. (2018). Metabolic analyses reveal different mechanisms of leaf color change in two purple-leaf tea plant (Camellia sinensis L.) cultivars. Horticulture Research, 5, 7, doi: 10.1038/ s41438-017-0010-1.

Shi, Y. L., Cai, Z. Y., Li, D., Lu, J. L., Ye, J. H., Liang, Y. R., AND ZhenG, X. Q. (2019). Effect of freezing on photosystem II and assessment of freezing tolerance of tea cultivar. Plants, 8(10), 434, doi: 10.3390/ plants 8100434.

Skrzyński, J., Leja, M., Gonkiewicz, A., And Banach, P. (2016). Cultivar effect on the sweet cherry antioxidant and some chemical attributes. Folia Horticulturae, 28(1), 95-102.

SzpadziK, E., Krupa, T., Niemiec, W., And JadczuKToBjasz, E. (2019). Yielding and fruit quality of selected sweet cherry (Prunus avium) cultivars in the conditions of central Poland. Acta Scientiarum Polonorum, Hortorum Cultus, 18, 117-126.

Wallace, T. C., And Giusti, M. M. (2019).Anthocyaninsnature's bold, beautiful, and health-promoting colors. Foods, 8(11), 550, doi:10.3390/foods 8110550.

Wang, L. X., Pan, D. Z., Liang, M., Abubakar, Y. S., Li, J., Lin, J. K., Chen, S. P., And Chen, W. (2017). Regulation of anthocyanin biosynthesis in purple leaves of Zijuan tea (Camellia sinensis var. kitamura). International Journal of Molecular Sciences, 18, 833, doi:10.3390/ijms18040833.

Wang, X., Liu, B. Y., ZhaO, Q., Sun, X., Li, Y., Duan, Z., Miao, X., Luo, S., And Li, J. (2019). Genomic variance and transcriptional comparisons reveal the mechanisms of leaf color affecting palatability and stressed defense in tea plant. Genes, 10(11), 929, doi: 10.3390/genes10110929.

Yang, L., Ling, W., Du, Z., Chen, Y., Li, D., Deng, S., LiU, Z., AND YANG, L. (2017). Effects of anthocyanins on cardiometabolic health: A systematic review and meta-analysis of randomized controlled trials. Advances in Nutrition, 8, 684-693.

YAnG, Y., ZhaO, Y., LiU, Z., AND LianG, G. Q. (2011). The third national regional test results of tea cultivars in Hunan Changsha. Tea Communication, 38(1), 3-7 (in Chinese).

Received: July 04, 2020; accepted: October 01, 2020. 